\title{
Application of a modified drop method for high-resolution pachytene chromosome spreads in two Phalaenopsis species
}

\author{
Yi-Tzu Kuo', Hui-Lan Hsu', Chih-Hsin Yeh² and Song-Bin Chang ${ }^{1 *}$
}

\begin{abstract}
Background: Preparation of good chromosome spreads without cytoplasmic contamination is the crucial step in cytogenetic mapping. To date, cytogenetic research in the Orchidaceae family has been carried out solely on mitotic metaphase chromosomes. Well-spread meiotic pachytene chromosomes can provide higher resolution and fine detail for analysis of chromosomal structure and are also beneficial for chromosomal FISH (fluorescence in situ hybridization) mapping. However, an adequate method for the preparation of meiotic pachytene chromosomes in orchid species has not yet been reported.

Results: Two Taiwanese native Phalaenopsis species were selected to test the modified drop method for preparation of meiotic pachytene chromosomes from pollinia. In this modified method, pollinia were ground and treated with an enzyme mixture to completely remove cell walls. Protoplasts were resuspended in ethanol/glacial acetic acid and dropped onto a wet inclined slide of $30^{\circ}$ from a height of $0.5 \mathrm{~m}$. The sample was then flowed down the inclined plane to spread the chromosomes. Hundreds of pachytene chromosomes with little to no cytoplasmic contamination were well spread on each slide. We also showed that the resolution of $45 \mathrm{~S}$ rDNA-containing chromosomes at the pachytene stage was up to 20 times higher than that at metaphase. Slides prepared following this modified drop method were amenable to FISH mapping of both $45 \mathrm{~S}$ and $5 \mathrm{~S}$ rDNA on pachytene chromosomes and, after FISH, the chromosomal structure remained intact for further analysis.

Conclusion: This modified drop method is suitable for pachytene spreads from pollinia of Phalaenopsis orchids. The large number and high-resolution pachytene spreads, with little or no cytoplasmic contamination, prepared by the modified drop method could be used for FISH mapping of DNA fragments to accelerate the integration of cytogenetic and molecular research in Phalaenopsis orchids.
\end{abstract}

Keywords: Phalaenopsis, Orchid, Pachytene chromosome, Fluorescence in situ hybridization (FISH), FISH mapping, Drop method

\section{Background}

Preparation of chromosome spreads is the foundation of cytogenetic research. Especially for the chromosomal mapping techniques, FISH (fluorescence in situ hybridization) or GISH (genomic in situ hybridization), well spread and intact chromosomes without interference from cytoplasm are critical to success. Additionally, the resolution of cytogenetic mapping depends on the degree of chromosome condensation. Compared with mitotic

\footnotetext{
* Correspondence: sbchang@mail.ncku.edu.tw

'Department of Life Sciences, National Cheng Kung University, No.1,

University Road, Tainan City 701, Taiwan

Full list of author information is available at the end of the article
}

metaphase chromosomes, the meiotic counterparts at the pachytene stage are much less condensed and reveal a clear distribution pattern of heterochromatic and euchromatic regions. Hence, using pachytene chromosomes greatly enhances the resolution of chromosome mapping and also benefits chromosome identification based solely on chromosomal morphology.

High-resolution FISH mapping on pachytene chromosomes is a powerful technique to help integrate physical and genetic maps and also to evaluate genome assembly quality. This strategy has been successfully demonstrated in plant species, for example tomato [1, 2], potato [3], cucumber [4], Rosa [5] and Amborella [6]. With the 
increasing use of sequencing, sequence datasets, such as transcriptomes [7, 8], BAC end sequences [9], and genomic shotgun sequences [10] have been generated for many orchid species, especially for Phalaenopsis orchids. However, molecular genetic information has not yet been integrated with cytogenetic information for these species.

Current chromosomal research in orchids, such as FISH mapping of repetitive sequences and rDNA in Dendrobium [11], Paphiopedilum [12] and Cymbidium [13] and also GISH analysis in Phalaenopsis [14] and Paphiopedilum [15], has mainly been carried out on mitotic metaphase chromosomes. Therefore, advanced research, like analysis of chromosomal structure and integration of orchid molecular and cytogenetic research through FISH mapping, might be restricted because of the poor resolution of metaphase chromosomes, especially for the orchid species with small chromosomes.

Certain natural characteristics of some orchid species, such as short flowering period, small number of flowers and small pollinia, limit material supply and further restrict chromosomal research on meiotic chromosomes. Further, the specialized pollinium, one of the main features of the Orchidaceae family, is composed of a large number of compactly gathered pollen mother cells (PMCs). The pollen masses enhance the difficulty in digesting the cell wall of PMCs evenly and preparing meiotic chromosome spreads.

The most common methods for preparing meiotic spreads are the squashing method $[16,17]$, the spreading method [18, 19] and the drop method [16, 20-23]. The squashing method was developed mainly for chromosome counting in plant species. The spreading method was reported to be suitable for plants with small chromosomes and has been applied in preparing mitotic chromosomes of rice, maize and tomato [18, 19, 24, 25]. The drop method has several advantages over the squashing method, despite the large quantity of materials required. Several slides with similar quality can be prepared from the same batch of cell suspension and chromosomes are more likely to spread apart from the cytoplasm. Without the interference of cytoplasm, the success of FISH or GISH mapping is improved. Moreover, both mitotic metaphase and meiotic pachytene chromosomes are often less distorted when prepared by the drop method compared with those prepared by the squashing method.

The spreading methods reported in the previous studies $[19,26,27]$ were successfully applied to prepare chromosome spreads for FISH mapping in tomato and Erycina pusilla in our laboratory. The pachytene chromosome spreads of Phalaenopsis species prepared following the reported protocols usually showed weak staining with DAPI or even lost after FISH. In addition, the conventional squashing method is incapable for preparing well-spread pachytene chromosomes from compactly gathered pollinia. Moreover, most previously reported drop methods were most solely applied in preparing mitotic metaphase chromosomes [21, 22], instead of less condensed and longer pachytene chromosomes. The recently developed 'SteamDrop' method was reported to be applicable in preparing both metaphase and pachytene chromosomes in wide range of species, however, technical expertise is demanded to get high-quality chromosome spreads [28].

In this study, we selected two Taiwanese native Phalaenopsis species as materials to develop and present the easily-mastered modified drop method for preparing high-resolution pachytene spreads. Additionally, the differences in chromosomal condensation level between chromosomes at the metaphase and pachytene stages in Phalaenopsis species were compared. Furthermore, 45S rDNA and 5S rDNA were mapped to demonstrate the applicability of the developed chromosome spreads in FISH mapping and analysis of chromosomal structure.

\section{Methods}

\section{Plant materials}

Two native Phalaenopsis species in Taiwan, $P$. aphrodite subsp. formosana $(2 \mathrm{n}=2 \mathrm{x}=38)$ and $P$. equestris $(2 \mathrm{n}=$ $2 x=38$ ), were selected as plant materials for preparation of chromosome spreads. Plants of both species were planted in a greenhouse and grown to flowering. Flower buds with size ranging from $8.50-9.20 \mathrm{~mm}$ and 4.50 $5.20 \mathrm{~mm}$ of $P$. aphrodite subsp. formosana and $P$. equestris, respectively, were collected to obtain developing pollinia undergoing meiosis with chromosomes at the pachytene stage. Root tips of $P$. aphrodite subsp. formosana were collected and treated with $2 \mathrm{mM} \mathrm{8-}$ Hydroxyquinoline at $15{ }^{\circ} \mathrm{C}$ for $3 \mathrm{~h}$. Pollinia and root tips were then fixed in freshly prepared Carnoy's fixative (glacial acetic acid: ethanol, 1:3 (v/v)) overnight at room temperature. After washing in distilled water for 5 min twice, they were stored in $70 \%$ ethanol at $-20{ }^{\circ} \mathrm{C}$ until use. A small piece of fixed pollinia was stained with $1 \%$ acetocarmine solution and was squashed immediately for examination of the chromosomal stage of PMCs.

\section{The modified drop method for preparation of meiotic pachytene spreads}

Pollinia with chromosomes at the pachytene stage were first rinsed in distilled water for 5 min twice and transferred to $10 \mathrm{mM}$ citrate buffer $(4 \mathrm{mM}$ citric acid and $6 \mathrm{mM}$ tri-sodium citrate, $\mathrm{pH}$ 4.5). The pollinia were dipped in $10 \mathrm{mM}$ citrate buffer, separated from anther cap and ground into small pieces using a tissue grinder in a microcentrifuge tube. The dispersed small pieces of pollinia were digested in enzyme mixture $(10 \mathrm{mM}$ citrate 
buffer containing $1 \%(\mathrm{w} / \mathrm{v})$ pectinase solution (Sigma), $1 \%(\mathrm{w} / \mathrm{v})$ pectolyase Y23 (Seishin Pharmaceutical) and $1 \%(\mathrm{w} / \mathrm{v})$ cellulose Onozuka RS (Yakult Pharmaceutical)) at $37^{\circ} \mathrm{C}$ for $30-45 \mathrm{~min}$ (P. aphrodite subsp. formosana) or 60-75 min (P. equestris). The sample was washed twice by adding distilled water to the digestion mixture, centrifuging for $12 \mathrm{~min}$ at 7,000 rpm, and discarding the supernatant. Distilled water was then substituted with freshly prepared Carnoy's fixative and the same centrifugation condition (7,000 rpm, $12 \mathrm{~min})$ and the substitution steps were repeated three times. The pellet was finally resuspended with $200 \mu \mathrm{l}$ Carnoy's fixative. The suspension was dropped onto a wet, acid-treated and inclined slide of $30^{\circ}$ from a height of $0.5 \mathrm{~m}$ using a sigmacote-treated glass dropper. The sample was then allowed to flow down the inclined plane to spread the chromosomes. After air drying, these slides were further fixed in Carnoy's fixative and then dehydrated in $95 \%$ ethanol for 3 min. Meiotic spreads with little to no cytoplasm and good pachytene chromosome spreading were selected and stored at $4{ }^{\circ} \mathrm{C}$ for later use.

\section{DNA probe preparation}

DNA fragments of 5S rDNA were amplified by PCR using forward primer 5S-F (5'-GAT CCC ATC AGA $\mathrm{ACT}$ TC-3') and reverse primer 5S-R (5'-GGT GCT TTA GTG CTG GTA T-3'). PCR was performed using $50 \mathrm{ng}$ genomic DNA, $100 \mu \mathrm{M}$ of dNTP, $0.2 \mu \mathrm{M}$ each of forward and reverse primer, $1 \times$ PCR buffer and $2.5 \mathrm{U}$ FastStart Taq DNA polymerase (Roche) in a total volume of $50 \mu \mathrm{l}$. The PCR cycling conditions followed the steps: $95{ }^{\circ} \mathrm{C}$ for $5 \mathrm{~min} ; 30$ cycles of $95{ }^{\circ} \mathrm{C} 30 \mathrm{~s}, 55^{\circ} \mathrm{C}$ for $2 \mathrm{~min}$ and $72{ }^{\circ} \mathrm{C}$ for $30 \mathrm{~s}$; final elongation at $72{ }^{\circ} \mathrm{C}$ for $5 \mathrm{~min}$. The DNA clone pTa71 containing $45 \mathrm{~S}$ rDNA of Triticum aestivum cloned into vector pAC184 was used to detect the position of 45S rRNA gene on pachytene chromosomes. Plasmid DNA was extracted using Plasmid Mini Prep Purification Kit (GMbiolab). The PCR product of 5S rDNA and plasmid DNA were labeled with either biotin-dUTP or digoxigenin-dUTP by standard nick translation following the manufacturer's protocol (Roche).

\section{Fluorescence in situ hybridization (FISH)}

FISH was carried out as previously described with some modifications [19]. The selected slides were incubated at $37{ }^{\circ} \mathrm{C}$ overnight or $65{ }^{\circ} \mathrm{C}$ for $30 \mathrm{~min}$ to air-dry the chromosome spreads before FISH. Pre-hybridization treatment included $5 \mu \mathrm{g} / \mathrm{ml}$ pepsin for $20 \mathrm{~min}$ and freshly prepared formaldehyde buffer (1× PBS, $50 \mathrm{mM}$ $\mathrm{MgCl}_{2}$ and $1 \%$ formaldehyde) for $10 \mathrm{~min}$. Slides were then dehydrated through an ethanol series (70, 90 and $100 \%)$. The hybridization mixture (10\% dextran sulfate sodium, $50 \%$ formamide, $2 \times$ SSC, $0.25 \%$ SDS and $100-200$ ng probe DNA) was boiled for $10 \mathrm{~min}$, placed on ice for 5-10 min and added onto the slides. The slides were treated at $80{ }^{\circ} \mathrm{C}$ for $2.5 \mathrm{~min}$ on a hot plate and incubated in a humid chamber at $37{ }^{\circ} \mathrm{C}$ for 12-16 h. Digoxigenin-labeled probes were detected with sheep fluorescein isothiocyanate (FITC)-conjugated anti-digoxigenin antibody (Roche) and amplified with anti-sheep-FITC (VECTOR Laboratories), whereas biotinlabeled probes were detected and amplified with Avidin Texas-red (VECTOR Laboratories) and Biotinylated Antiavidin D (VECTOR Laboratories), respectively. Finally, slides were dehydrated through an ethanol series and dried at $37{ }^{\circ} \mathrm{C}$ for $20 \mathrm{~min}$. Chromosomes were counterstained with $1.5 \mu \mathrm{g} / \mathrm{ml}$ 4; 6-diamidino-2-phenylindole (DAPI) in mounting medium (VECTOR Laboratories).

\section{Image capturing and cytological analysis}

Images were captured using a Nikon DS Ri1 CCD camera attached to a Nikon ECLIPSE 80i microscope. Image adjustment and chromosomal straightening were carried out using ImageJ software. The length of the chromosome and the distance from the signal to the end of the chromosome were measured using NIS-Elements microscope imaging software (Nikon). The relative distance to the signal was calculated by the formula: Distance from the end of the short arm to the signal $\times 100 \% /$ length of the pachytene chromosome.

\section{Results}

Collection of flower buds and examination of chromosome stage in PMCs

Flower buds, with sizes ranging from $8.50-9.20 \mathrm{~mm}$ for P. aphrodite subsp. formosana and $4.50-5.20 \mathrm{~mm}$ for $P$. equestris, were examined, with most PMCs having mostly meiotic chromosomes at the pachytene stage (Fig. 1a-b). However, PMCs with chromosomes at the zygotene or diakinesis stages were also observed in these flower buds. Most of the flower buds of $P$. aphrodite subsp. formosana with size over $9.50 \mathrm{~mm}$ had PMCs at metaphase I or even at the tetrad stage (Fig. 1c-d). For Phalaenopsis species, pollinia contain two halves of the pollinium attached to an anther cap. Sometimes asynchronous cell division of PMCs between the two halves of the pollinium was observed.

\section{Preparation of meiotic chromosome spreads using the modified drop method}

Owing to the loss of some PMCs during chromosome preparation, particularly in protoplast preparation, pollinia from one $P$. aphrodite subsp. formosana flower bud or 2-3 pollinia of $P$. equestris were used to prepare 1525 slides with similar quality. A tissue grinder was used to crush masses of PMCs separated from the anther cap and also to disperse pollinia into uniformly small pieces 

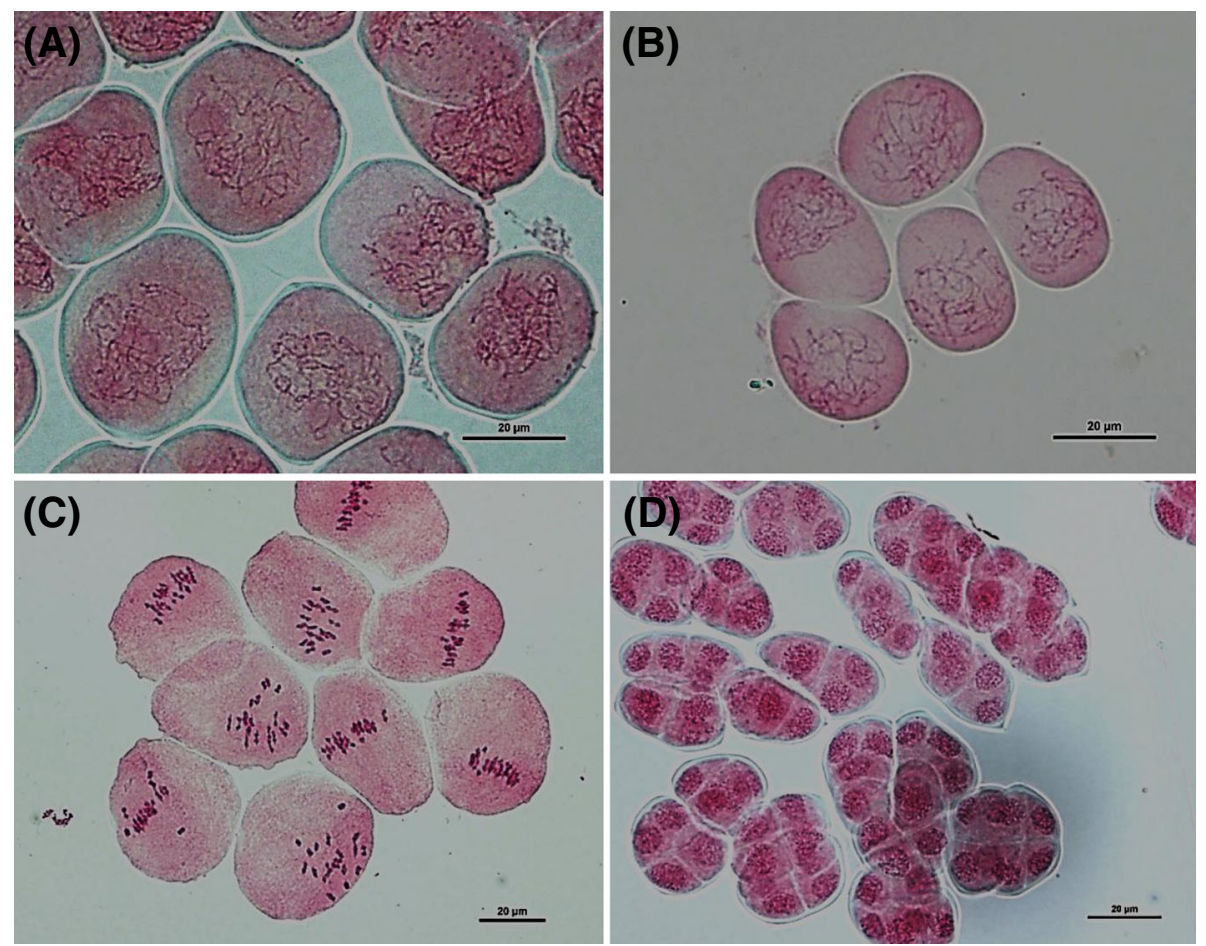

Fig. 1 Examination of meiotic chromosome stage in pollen mother cells (PMCs). Pachytene stage in P. aphrodite subsp. formosana (a) and in P. equestris (b). Chromosomes at prometaphase I, metaphase I (c) and tetrad stages (d) of PMCs from P. aphrodite subsp. formosana. PMCs were stained with $1 \%$ acetocarmine, scale bar $=20 \mu \mathrm{m}$

in a microcentrifuge tube. To eliminate the cell wall of PMCs, the same enzyme mixture was applied in the two species. The optimal enzyme treatment incubation was found to be 30-45 min and 60-75 min for P. aphrodite subsp. formosana and $P$. equestris, respectively. After such treatment, over $80 \%$ (visual observation) of meiotic spreads were free from interference of wall fragments and cytoplasm. Slight interference from the cytoplasm might have no effect on further chromosome staining but pre-treatment with pepsin might be needed before FISH hybridization to facilitate probe penetration. The cell suspensions of the two Phalaenopsis species were dropped onto wet slides from a height of $0.5 \mathrm{~m}$ and most of the pachytene chromosomes were structurally intact and also well spread (Fig. 2a-b). Approximately 100-250 meiotic pachytene spreads were obtained on each slide following this modified drop method. Converting images of DAPI-stained pachytene chromosomes into black-and-white with a white background enhanced the visual contrast between heterochromatic and euchromatic regions (Fig. 2c). However, brightly stained chromomeres on some pachytene chromosomes of $P$. aphrodite subsp. formosana were easily observed in an image with a black background (Fig. 2d).

Meiotic spreads at stages other than pachytene, like metaphase I, can also be prepared using this method.
Nineteen non-overlapping tetrads of $P$. equestris (Fig. 2e) and P. aphrodite subsp. formosana (Fig. 2f) at metaphase I are shown. Adjustment of the dropping height might avoid overlap of the highly condensed chromosomes.

\section{FISH mapping of 45S rDNA and comparison of chromosome condensation at metaphase and pachytene stages}

The basic chromosome number of Phalaenopsis species is up to $\mathrm{x}=19$. Therefore, it is relatively hard to trace and identify every chromosome in a single cell, especially the more tangled pachytene chromosomes, and to measure the total complement length. To compare the levels of chromosome condensation at different stages, $45 \mathrm{~S}$ rDNA was mapped on both metaphase and pachytene chromosomes of $P$. aphrodite subsp. formosana using FISH, and the length of this $45 \mathrm{~S}$ rDNA-containing chromosome was measured. First, the applicability of chromosome spreads prepared by this modified drop method to FISH mapping was proved. Second, a hybridization signal of $45 \mathrm{~S}$ rDNA was detected on one end of a meiotic pachytene chromosome (Fig. 3a) and a pair of signals was observed on two mitotic metaphase chromosomes in P. aphrodite subsp. formosana (Fig. 3b). $45 \mathrm{~S}$ rDNA is a highly repetitive locus in the plant genome. A strong signal for $45 \mathrm{~S}$ rDNA was observed 


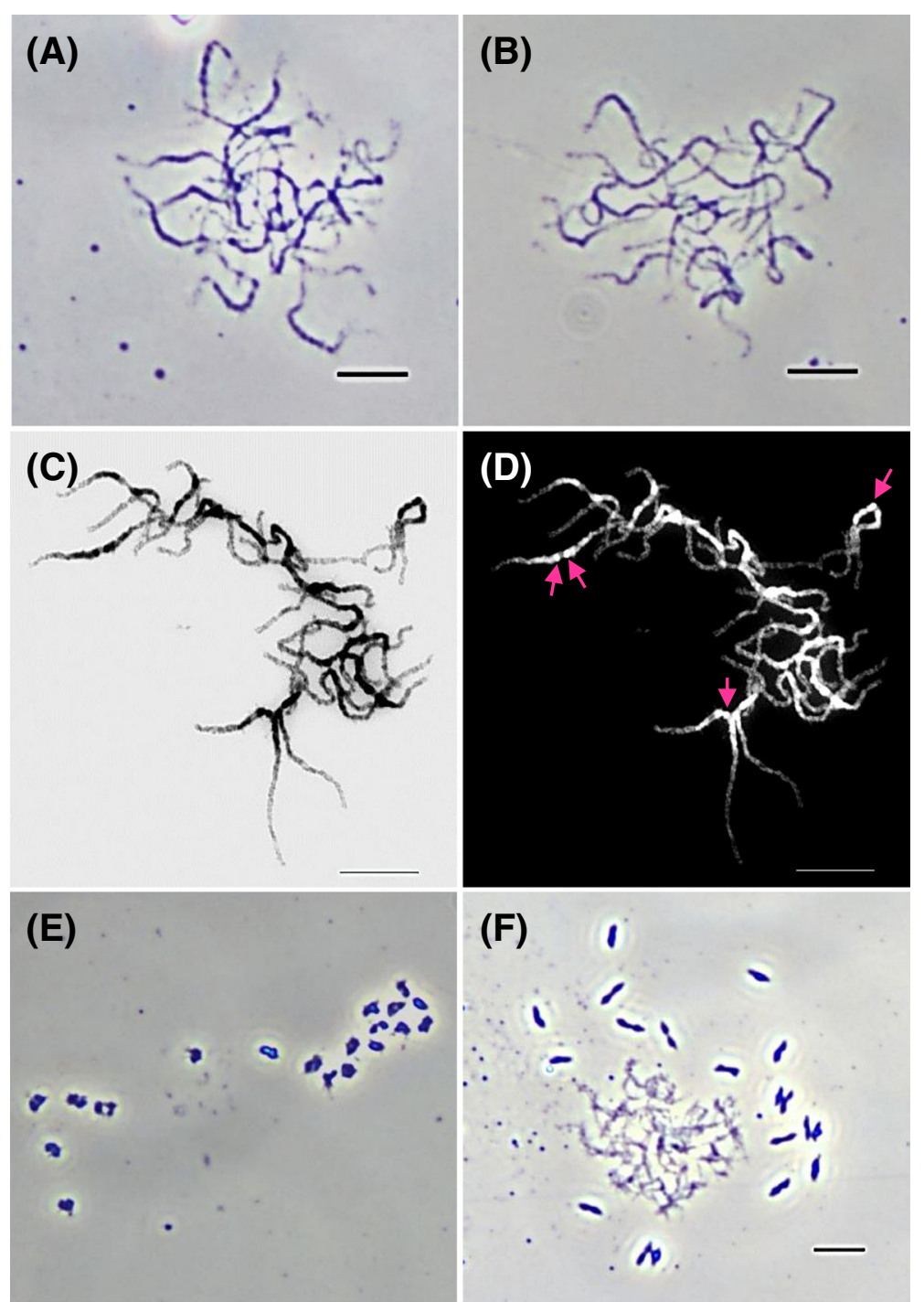

Fig. 2 Phalaenopsis meiotic spreads prepared by the modified drop method. Pachytene spread of $P$. equestris (a) and P. aphrodite subsp. formosana (b). c-d The DAPI-staining pachytene chromosomes of $P$. aphrodite subsp. formosana were converted to black and white to enhance the visualization of chromosomal structure. The pink arrows in (d) indicate chromomeres on pachytene chromosomes. Chromosomes of $P$. equestris (e) and P. aphrodite subsp. formosana (f) at meiotic metaphase I. Scale bar $=10 \mu \mathrm{m}$

and can be detected on almost every chromosome spread prepared by this method. Fourteen $45 \mathrm{~S}$ rDNAcontaining pachytene chromosomes were straightened; their length ranged from $19.76 \mu \mathrm{m}$ to $25.84 \mu \mathrm{m}$ (average $22.06 \mu \mathrm{m}$ ), while the length of this chromosome at metaphase was only about $1.05 \mu \mathrm{m}$ (Fig. 3c). This result indicated that chromosomes at the meiotic pachytene stage provide over 20 times the resolution of those at mitotic metaphase in $P$. aphrodite subsp. formosana. We were also able to observe that most of the $45 \mathrm{~S}$ rDNAcontaining chromosome was euchromatin except for the bright DAPI-staining pericentromeric heterochromatin and the NOR (nucleolus organizer region) on the end of the short arm (Fig. 3c). This demonstrates that the distribution pattern of heterochromatin and euchromatin on pachytene spreads prepared by this method can be clearly distinguished by DAPI staining.

\section{Precise location of $5 \mathrm{~S}$ rDNA on pachytene chromosomes}

The 5S rDNA of both $P$. aphrodite subsp. formosana and $P$. equestris was amplified and mapped on pachytene chromosomes by FISH to show its precise location. Obvious signals of $5 \mathrm{~S}$ rDNA were detected on two pachytene chromosomes of $P$. aphrodite subsp. formosana (Fig. 4a) and only one signal presented in P. equestris pachytene chromosomes (Fig. 4b). Each of these 5S rDNA-containing pachytene chromosomes were traced and straightened. The signals of $5 \mathrm{~S}$ rDNA were all 


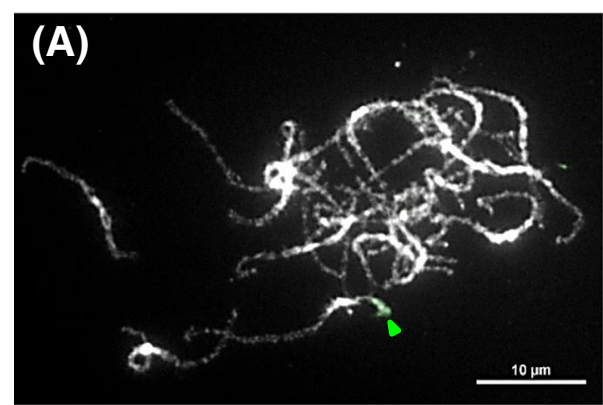

(C)
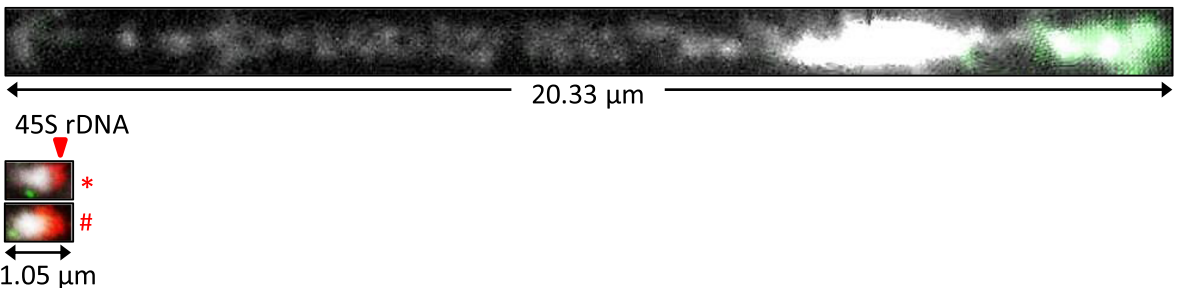

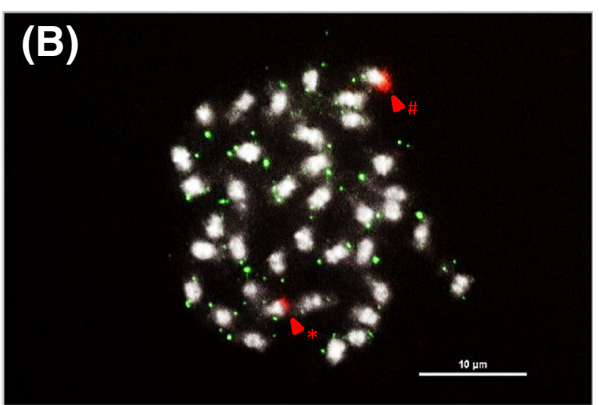

$45 \mathrm{~S}$ rDNA

$$
1.05 \mu \mathrm{m}
$$

Fig. 3 FISH mapping of $45 \mathrm{~S}$ rDNA (arrowheads) on chromosomes at pachytene stage (a) and metaphase (b) in P. aphrodite subsp. formosana. Telomere DNA in green (b) was also mapped on metaphase chromosomes. $\mathbf{c}$ The pachytene and metaphase chromosomes with 45S rDNA signals were straightened using ImageJ software. The asterisk and pound sign indicate the corresponding chromosomes in (b). Chromosomes were stained with DAPI and images were converted to black and white. Scale bar $=10 \mu \mathrm{m}$
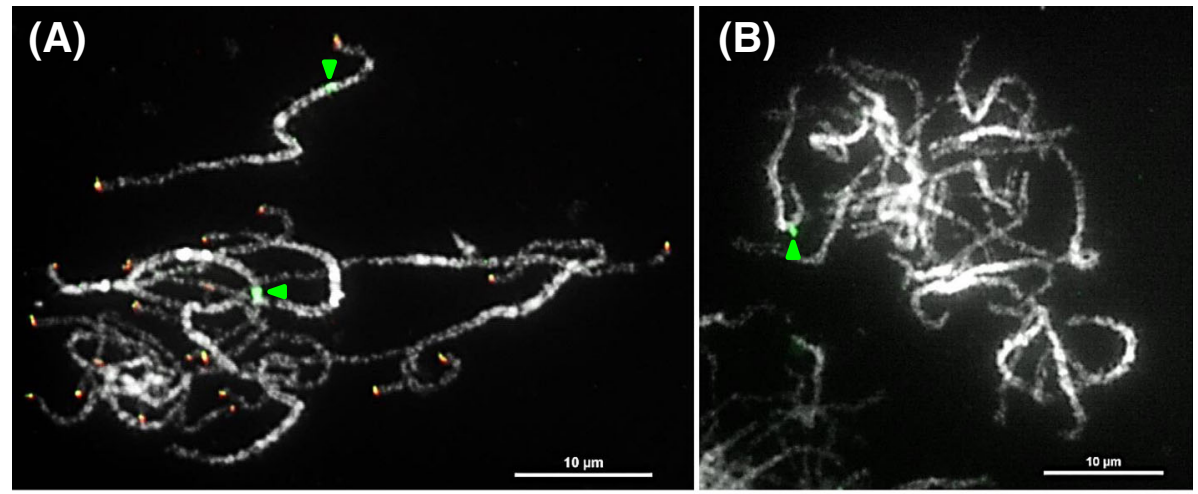

(C)

$\nabla(23.27 \%)$
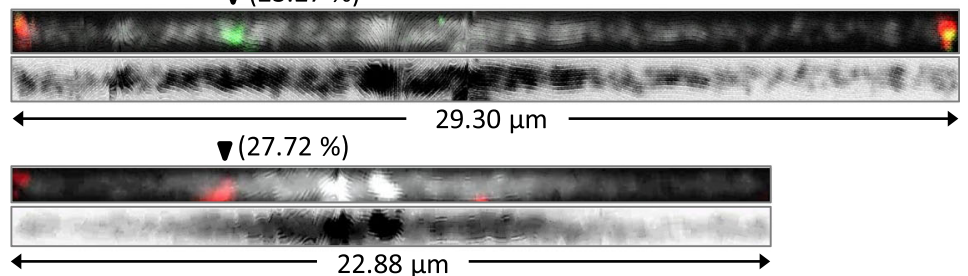

(D)

( $(23.11 \%)$

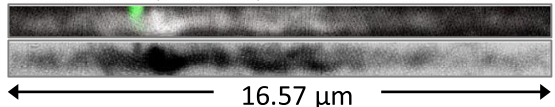

Fig. 4 FISH mapping of $5 S$ rDNA on pachytene chromosomes. a Two distinct signals of 55 rDNA (green arrowheads) were observed in P. aphrodite subsp. formosana and the red signals represent telomeres. b Only one signal of $5 \mathrm{~S}$ rDNA (green arrowhead) was detected in P. equestris. The 55 rDNAlocated chromosomes of $P$. aphrodite subsp. formosana (c) and $P$. equestris (d) were straightened using ImageJ and arrowheads indicated the signals of $5 S$ rDNA. The relative distance of a signal on a pachytene chromosome was shown as a percentage. The images of DAPI-stained chromosomes were converted to black and white. Scale bar $=10 \mu \mathrm{m}$ 
mapped on the short arm of these chromosomes. The relative distances of the $5 \mathrm{~S}$ rDNA signals on the two pachytene chromosomes of $P$. aphrodite subsp. formosana were 23.27 and $27.72 \%$ (Fig. 4c) and the relative distance of the signal in $P$. equestris was $23.11 \%$ (Fig. 4d). Noticeably, the two 5S rDNA-containing chromosomes of $P$. aphrodite subsp. formosana can be easily discriminated owing to the presence of two obvious chromomeres on one of these two chromosomes (Fig. 4c). In summary, the pachytene spreads prepared by the modified drop method can be applied in FISH mapping, with chromosomal structure remaining intact after FISH for further analysis.

\section{Discussion}

\section{Advantages of the modified drop method}

The drop method was first developed for preparing chromosome spreads of human cells [29] and for protoplast isolation in plant species [30]. Since then, several methods have been published to improve the techniques of meiotic chromosome preparation for plant species $[19,28,31,32]$, but none of these methods has been reported suitable for orchid species with specialized pollinia. In this study, we developed the first easily mastered drop method for preparation of high-quality meiotic pachytene chromosomes in Phalaenopsis species. The protocol of drop preparation of plant chromosomes [33] combined with the other two reported methods $[34,35]$ were taken as the original protocols to develop the modified drop method. In this method, tissue grinders are used to crush specialized Phalaenopsis pollinia into scattered PMCs for eliminating cell walls completely. Repeat times of centrifugation/resuspension and speed of centrifugation are optimized to decrease the amount of dirt in suspension and avoid excessive sample loss. The adequate dropping height is adjusted to bombard the cell suspension onto the wet slides for Phalaenopsis species. The samples then flow down freely along the inclined plane to spread chromosomes, instead of blowing or spraying bursting medium, which is relatively more technical expertise demanded. Therefore, the advantages of this method over the previous methods include: (1) the cell walls of tightly compacted PMCs can be uniformly eliminated; (2) no technical expertise is needed for dropping protoplasts onto inclined slides using a dropper, or spreading chromosomes; (3) most chromosome spreads are free from contamination by wall fragments and cytoplasm; (4) several slides with similar quality can be prepared in a single experiment; (5) up to 100 meiotic pachytene spreads can be obtained on each slide; (6) the chromosomal structure remains intact even after FISH when samples are dropped from the optimized dropping height. However, different numbers of pollinia may be required from different species to obtain sufficient PMCs for spread preparation following this protocol. In Phalaenopsis species like P. equestris, two to three pollinia were required to prepare slides with a similar spread number to $P$. aphrodite subsp. formosana using pollinia from only one flower bud. Additionally, asynchronous cell divisions of PMCs in the two halves of the pollinium were sometimes found. Hence, examining the chromosomal stage of PMCs in both halves of the pollinium, before preparation of meiotic chromosome spreads, is recommended to avoid getting chromosome spreads at different division stages on the same slide.

\section{High resolution of meiotic pachytene chromosomes of Phalaenopsis species}

The length differences of chromosome counterparts at pachytene and metaphase stages among different plant species are diverse. The chromosomes at the pachytene stage are much longer than those at metaphase, with a 7-fold length difference for rye, 10-fold for maize, 15fold for tomato, over 20-fold for Arabidopsis and up to 40-fold for rice [36]. According to the length analysis of the 45S rDNA-containing chromosome, the length of the chromosomes at meiotic pachytene was over 20 -fold longer than that at mitotic metaphase in $P$. aphrodite subsp. formosana. A pair of 5S rDNA signals was detected on the end of two mitotic metaphase chromosomes in P. equestris [37]. While mapping of $5 \mathrm{~S}$ rDNA revealed one signal on the meiotic pachytene chromosomes, our result showing only one $5 \mathrm{~S}$ rDNA locus in $P$. equestris genome was consistent with previous studies. However, the relative distance of $5 \mathrm{~S}$ rDNA signal on the chromosome at the pachytene stage was $23.27 \%$, not on the end as is suggested by analysis of metaphase chromosomes. This demonstrates that the higher resolution of the meiotic pachytene chromosome makes it the stage of choice to detect the precise location of DNA fragments on chromosomes. Moreover, pachytene spreads of $P$. aphrodite subsp. formosana prepared by the modified drop method preserved obvious chromomere structures on certain chromosomes, including two chromomeres on one of the $5 \mathrm{~S}$ rDNA-containing chromosomes. The chromomeres on pachytene chromosomes can be taken as unique markers for chromosome identification. Together these results suggest that preparation of Phalaenopsis pachytene chromosomes, with higher resolution and obvious structural characteristics, will benefit further cytogenetic research in Phalaenopsis species.

\section{Further applicable prospects of this modified drop method}

The first draft genome sequence of the Orchidaceae family was determined in $P$. equestris [10], but the 
assembled scaffolds have not yet been mapped to chromosomes or linkage groups. Cytogenetic studies of Phalaenopsis species are relatively rare and karyotypes of the few Phalaenopsis species investigated were constructed based solely on metaphase chromosomes [38]. Highresolution pachytene chromosomes have not been prepared and investigated in Phalaenopsis cytogenetic research. High-quality pachytene spreads can be easily prepared using this modified drop method and welldispersed pachytene chromosomes are amenable for establishing high-resolution karyotypes. The pachytene chromosome-based karyotype has been established in several species like cucumber [39], tomato [27], potato [3], papaya [40] and Daucus species [41], but not yet in Phalaenopsis species. Slides with large numbers of pachytene spreads combined with well-developed FISH mapping techniques are suitable for large-scale cytogenetic analysis to help verify the quality of genome assembly and integrate physical and genetic maps. This modified drop method could be further used to prepare meiotic chromosome spreads from pollinia of orchid species other than Phalaenopsis species, although the conditions of dropping height and enzyme treatment for different species, or preparing spreads of chromosomes at different stages must be optimized. Moreover, laser capture microdissection is an increasingly popular technique to isolate specific chromosomal regions or DNA fragments spread on membrane-coated slides [42]. The modified drop method could also be used to prepare chromosome spreads on membrane-coated slides, with less contamination and therefore less interference in downstream applications such as DNA amplification.

\section{Conclusions}

Here we describe and demonstrate for the first time a modified drop method for the preparation of pachytene chromosome spreads from the specialized pollinia in Phalaenopsis orchids. The up to 20 -fold higher resolution of chromosomes at the pachytene, compared with the metaphase, stage, provides a marked improvement in the information that can be acquired using FISH mapping, resulting in improved cytogenetic investigations in Phalaenopsis species. In addition to the high resolution, the chromosome spreads prepared by this method can reveal clear patterns of heterochromatic and euchromatic regions and obvious chromomere structures. The applicability of the prepared chromosome spreads was also demonstrated by FISH mapping of both $45 \mathrm{~S}$ and $5 \mathrm{~S}$ rDNA. The signals of these rDNA probes were easily detected and the chromosomal structure after FISH remained intact for further analysis. Moreover, 15-25 slides of similar quality can be prepared from the same batch of cell suspension and 100-250 pachytene spreads can be obtained on one slide using this method. Combining the high-quality pachytene chromosomes with well-established FISH mapping techniques will result in a powerful tool for large-scale cytogenetic research, and will further accelerate the integration of molecular and cytogenetic research in Phalaenopsis and other orchid species.

\section{Abbreviations \\ DAPI, 4', 6-diamidino-2-phenylindole; FISH, fluorescence in situ hybridization; FITC, fluorescein isothiocyanate; GISH, genome in situ hybridization; NOR, nucleolus organizer region; PBS, phosphate buffered saline; PMC, pollen mother cell; rDNA, ribosomal DNA; SDS, sodium docecyl sulfate; SSC, saline sodium citrate}

\section{Acknowledgements}

This study is supported by Innovative Translational Agricultural Research under Grand No. 2016AG001 from Academia Sinica, Taiwan and by Ministry of Science and Technology (MOST)/National Science Council, Taiwan under Grant No. NSC 99-2313-B-002-003-MY3.

\section{Availability of data and materials}

The information supporting the conclusions of this article is included within the article and more details are available on request. Please contact the corresponding author through email.

\section{Authors' contributions}

Modification of drop method and chromosome preparation was conducted by YTK and HLH. FISH was carried by YTK, assisted by HLH. The plant materials were provided and harvested by CHY. YTK wrote the manuscript. All processes were coordinated and guided by SBC. All authors read and approved the final manuscript.

\section{Competing interests}

The authors declare that they have no competing interests.

\section{Author details}

'Department of Life Sciences, National Cheng Kung University, No.1, University Road, Tainan City 701, Taiwan. ${ }^{2}$ Taoyuan District Agricultural Research and Extension Station, Council of Agriculture, Executive Yuan, 139, Sec. 2, Dongfu Rd., Houzhuang, Sinwu Dist., Taoyuan City 32745, Taiwan.

Received: 21 April 2016 Accepted: 2 June 2016

Published online: 06 June 2016

\section{References}

1. Szinay D, Chang SB, Khrustaleva L, Peters S, Schijlen E, Bai Y, Stiekema WJ, van Ham RC, de Jong H, Klein Lankhorst RM. High-resolution chromosome mapping of BACs using multi-colour FISH and pooled-BAC FISH as a backbone for sequencing tomato chromosome 6. Plant J. 2008;56(4):627-37.

2. Shearer LA, Anderson LK, de Jong H, Smit S, Goicoechea JL, Roe BA, Hua A, Giovannoni JJ, Stack SM. Fluorescence in situ hybridization and optical mapping to correct scaffold arrangement in the tomato genome. G3-Genes Genom Genet. 2014;4(8):1395-405.

3. lovene M, Wielgus SM, Simon PW, Buell CR, Jiang J. Chromatin structure and physical mapping of chromosome 6 of potato and comparative analyses with tomato. Genetics. 2008;180(3):1307-17.

4. Lou Q, He Y, Cheng C, Zhang Z, Li J, Huang S, Chen J. Integration of highresolution physical and genetic map reveals differential recombination frequency between chromosomes and the genome assembling quality in cucumber. PLoS One. 2013;8(5):e62676.

5. Kirov IV, Van Laere K, Khrustaleva LI. High resolution physical mapping of single gene fragments on pachytene chromosome 4 and 7 of Rosa. BMC Genet. 2015;16:74

6. Chamala S, Chanderbali AS, Der JP, Lan T, Walts B, Albert VA, de Pamphilis CW Leebens-Mack J, Rounsley S, Schuster SC, et al. Assembly and validation of the genome of the nonmodel basal angiosperm Amborella. Science. 2013;342(6165):1516-7. 
7. Su CL, Chao YT, Alex Chang YC, Chen WC, Chen CY, Lee AY, Hwa KT, Shih MC. De novo assembly of expressed transcripts and global analysis of the Phalaenopsis aphrodite transcriptome. Plant Cell Physiol. 2011;52(9):1501-14.

8. Chao YT, Su CL, Jean WH, Chen WC, Chang YC, Shih MC. Identification and characterization of the microRNA transcriptome of a moth orchid Phalaenopsis aphrodite. Plant Mol Biol. 2014;84(4-5):529-48.

9. Hsu CC, Chung YL, Chen TC, Lee YL, Kuo YT, Tsai WC, Hsiao YY, Chen YW, Wu WL, Chen HH. An overview of the Phalaenopsis orchid genome through BAC end sequence analysis. BMC Plant Biol. 2011;11:3.

10. Cai J, Liu X, Vanneste K, Proost S, Tsai WC, Liu KW, Chen L, He Y, Xu Q, Bian C, et al. The genome sequence of the orchid Phalaenopsis equestris. Nat Genet. 2015;47(1):65-72.

11. Begum R, Alam SS, Menzel G, Schmidt T. Comparative molecular cytogenetics of major repetitive sequence families of three Dendrobium species (Orchidaceae) from Bangladesh. Ann Bot-London. 2009;104(5):863-72.

12. Lan TY, Albert VA. Dynamic distribution patterns of ribosomal DNA and chromosomal evolution in Paphiopedilum, a lady's slipper orchid. BMC Plant Biol. 2011;11:126.

13. Sharma SK, Mehra P, Kumari J, Kumar S, Kumaria S, Tandon P, Rao SR. Physical localization and probable transcriptional activity of 18S-5.8 S-26S rRNA gene loci in some Asiatic Cymbidiums (Orchidaceae) from north-east India. Gene. 2012;499(2):362-6.

14. Lin C, Chen Y, Chen W, Chen C, Kao Y. Genome organization and relationships of Phalaenopsis orchids inferred from genomic in situ hybridization. Bot Bull Acad Sin. 2005;46:339-45.

15. Lee YI, Chung MC. Identification of genome relationships among Paphiopedilum species by genomic and fluorescent in situ hybridization. Acta Hortic. 2008;766:331-4.

16. Schwarzacher T, Leitch AR. Enzymatic treatment of plant material to spread chromosomes for in situ hybridization. Protoc Nucleic Acid Anal Nonradioactive Probes. 1994;28:153-60.

17. Schwarzacher T, Ambros P, Schweizer D. Application of Giemsa-banding to orchid karyotype analysis. Plant Syst Evol. 1980;134(3-4):293-7.

18. Fukui K, lijima K. Somatic chromosome map of rice by imaging methods. Theor Appl Genet. 1991;81(5):589-96.

19. Zhong $\mathrm{XB}$, de Jong JH, Zabel P. Preparation of tomato meiotic pachytene and mitotic metaphase chromosomes suitable for fluorescence in situ hybridization (FISH). Chromosome Res. 1996;4(1):24-8.

20. Kato A, Lamb JC, Birchler JA. Chromosome painting using repetitive DNA sequences as probes for somatic chromosome identification in maize. Proc Natl Acad Sci U S A. 2004;101(37):13554-9.

21. Kato A. Air drying method using nitrous oxide for chromosome counting in maize. Biotech Histochem. 1999;74(3):160-6.

22. Aliyeva-Schnorr L, Ma L, Houben A. A fast air-dry dropping chromosome preparation method suitable for FISH in plants. J Vis Exp. 2015;106:e53470.

23. Kato A, Lamb JC, Albert PS, Danilova T, Han F, Gao Z, Findley S, Birchler JA. Chromosome painting for plant biotechnology. Methods Mol Biol. 2011;701:67-96

24. Decarvalho CR, Saraiva LS. An air drying technique for maize chromosomes without enzymatic maceration. Biotech Histochem. 1993;68(3):142-5.

25. Andras SC, Hartman TPV, Marshall JA, Marchant R, Power JB, Cocking EC, Davey MR. A drop-spreading technique to produce cytoplasm-free mitotic preparations from plants with small chromosomes. Chromosome Res. 1999;7(8):641-7.

26. Yeh HY, Lin CS, Chang SB. Cytogenetic and cytometric analyses in artificial intercytotypic hybrids of the emergent orchid model species Erycina pusilla. Euphytica. 2015;206(2):533-9.

27. Chang SB, Yang TJ, Datema E, van Vugt J, Vosman B, Kuipers A, Meznikova M, Szinay D, Lankhorst RK, Jacobsen E, et al. FISH mapping and molecular organization of the major repetitive sequences of tomato. Chromosome Res. 2008;16(7):919-33.

28. Kirov I, Divashuk M, Van Laere K, Soloviev A, Khrustaleva L. An easy "SteamDrop" method for high quality plant chromosome preparation. Mol Cytogenet. 2014;7:21.

29. Rothfels $K H$, Siminovitch L. An air-drying technique for flattening chromosomes in mammalian cells grown in vitro. Stain Technol. 1958;33(2):73-7.

30. Murata M. Staining air dried protoplasts for study of plant chromosomes. Stain Technol. 1983;58(2):101-6.

31. Dang JB, Zhao Q, Yang X, Chen Z, Xiang SQ, Liang GL. A modified method for preparing meiotic chromosomes based on digesting pollen mother cells in suspension. Mol Cytogenet. 2015;8:80
32. Iacia AAS, Pinto-Maglio CAF: Mapping pachytene chromosomes of coffee using a modified protocol for fluorescence in situ hybridization. Aob Plants 2013;5:plt040.

33. Schwarzacher T, Heslop-Harrison P. Practical in situ hybridization. Oxford: BIOS; 2000. p. 60-1.

34. Peterson DG, Price HJ, Johnston JS, Stack SM. DNA content of heterochromatin and euchromatin in tomato (Lycopersicon esculentum) pachytene chromosomes. Genome. 1996;39(1):77-82.

35. Chang SB, Anderson LK, Sherman JD, Royer SM, Stack SM. Predicting and testing physical locations of genetically mapped loci on tomato pachytene chromosome 1. Genetics. 2007:176(4):2131-8.

36. de Jong JH, Fransz P, Zabel P. High resolution FISH in plants - techniques and applications. Trends Plant Sci. 1999;4(7):258-63.

37. Lee YH, Kao YY. Distribution of ribosomal RNA genes in seven Phalaenopsis species. Taipei: Institute of Molecular and Cellular Biology, National Taiwan University; 2006. Master Thesis.

38. Kao YY, Chang SB, Lin TY, Hsieh CH, Chen YH, Chen WH, Chen CC. Differential accumulation of heterochromatin as a cause for karyotype variation in Phalaenopsis orchids. Ann Bot-London. 2001;87(3):387-95.

39. Koo DH, Choi HW, Cho J, Hur Y, Bang JW. A high-resolution karyotype of cucumber (Cucumis sativus L. 'Winter Long') revealed by C-banding, pachytene analysis, and RAPD-aided fluorescence in situ hybridization. Genome. 2005;48(3):534-40.

40. Zhang W, Wai CM, Ming R, Yu Q, Jiang J. Integration of genetic and cytological maps and development of a pachytene chromosome-based karyotype in papaya. Trop Plant Biol. 2010;3(3):166-70.

41. Iovene M, Cavagnaro PF, Senalik D, Buell CR, Jiang JM, Simon PW Comparative FISH mapping of Daucus species (Apiaceae family). Chromosome Res. 2011;19(4):493-506.

42. Schermelleh L, Thalhammer S, Heckl W, Posl H, Cremer T, Schutze K, Cremer M. Laser microdissection and laser pressure catapulting for the generation of chromosome-specific paint probes. Biotechniques. 1999;27(2):362-7.

\section{Submit your next manuscript to BioMed Central and we will help you at every step:}

- We accept pre-submission inquiries

- Our selector tool helps you to find the most relevant journal

- We provide round the clock customer support

- Convenient online submission

- Thorough peer review

- Inclusion in PubMed and all major indexing services

- Maximum visibility for your research

Submit your manuscript at www.biomedcentral.com/submit
C Biomed Central 